\title{
De l'usage de la parole proverbiale dans les textes dramatiques
}

André Petitjean et Sabine Petillon

\section{(2) OpenEdition}

1 Journals

\section{Édition électronique}

URL : http://journals.openedition.org/pratiques/2880

DOI : 10.4000/pratiques.2880

ISSN : 2425-2042

Éditeur

Centre de recherche sur les médiations (CREM)

\section{Édition imprimée}

Date de publication : 15 décembre 2013

\section{Référence électronique}

André Petitjean et Sabine Petillon, « De l'usage de la parole proverbiale dans les textes dramatiques », Pratiques [En ligne], 159-160 | 2013, mis en ligne le 30 juin 2016, consulté le 14 novembre 2019. URL http://journals.openedition.org/pratiques/2880; DOI : 10.4000/pratiques.2880

\section{(c) Tous droits réservés}




\title{
De l'usage de la parole proverbiale dans les textes dramatiques
}

\author{
André Petitjean \\ Université de Lorraine, CREM \\ Sabine Petillon \\ C.N.R.S/ Modyco - Université Paris Ouest Paris X
}

\section{Introduction}

Dans le cadre de ce numéro de Pratiques consacré au figement, nous avons choisi d'étudier la parole proverbiale dans les dialogues théâtraux. Dans une première partie ( «Statut de la parole proverbiale»), nous ferons un bref examen des théories linguistiques consacrées aux proverbes. Dans une seconde partie («Énonciation proverbiale et dialogisme»), nous décrirons les modes d'insertion des proverbes dans les échanges dialogués (statut du locuteur premier, modalités de l'insertion de la citation).Dans une troisième partie, nous étudierons les fonctions textuelles des proverbes au sein de l'économie interne des textes dramatiques, selon qu'ils correspondent à des proverbes attestés ou réécrivent des proverbes connus.

\section{Statut de la parole proverbiale}

Qu'elles aient pour origine des folkloristes, des ethnologues ou des linguistes, les études parémiologiques sont loin d'être consensuelles quand elles cherchent à définir les faits sentencieux. Pour certains (P.J.L. Arnaud, 1991), il est pertinent d'opposer, d'un côté, les maximes, les préceptes, les apophtegmes, les aphorismes ou les devises, et, de l'autre, les proverbes, les dictons, les adages etc. Les premiers seraient perçus comme ayant un énonciateur individué et spécifique, sous la forme d'un auteur connu et reconnu, à l'origine d'une « parole ailée »: 
L'AVOCAT : [...] et c'est fort à propos que qu'Hypocrate dit dans son premier aphorisme : Vita brevis, ars vero longa, occasio praeceps, experimentum periculosum, judicium difficile.

(Molière, Le Médecin volant, scène VIII, Gallimard, "Bibliothèque de La

Pléiade », tome 1, p. 37)

MARIANA. [...] Il n'y a nulle affectation en mon père. Il va droit devant lui. Son salut propre, et l'Ordre, voilà sa voie : à droite et à gauche, rien. Son indifférence écrasante pour tout ce qui ne porte pas quelque marque sublime... Unum, Domine, "O mon dieu! une seule chose est nécessaire » : mon grand-père savait ce qu'il faisait, lorsqu'il changeait en cette devise-là la devise plus antique de notre famille.

(Montherlant, Le Maître de Santiago, Acte II, scène 3, Livre de poche, ${ }^{\circ} 1172$, p. 99)

Pour les seconds, l'énonciateur premier, qui doit bien exister, est indéfini et non spécifique. C'est le fameux «ON-locuteur», caractérisé comme étant «la sagesse des nations », la « sagesse populaire », l'« opinion publique » ou « l'observation quotidienne $»$ :

ALVARO. [...] La trahison est toujours sous notre toit, et pas seulement à la cuisine, comme on le dit. (Appelant:) Mariana.

(Montherlant, Le Maître de Santiago, Acte II, scène 1, Livre de poche, ${ }^{\circ}{ }^{1172,}$ p. 90)

En fait cette opposition n'est pas unanimement partagée (J.-C. Anscombre (1994) la récuse) et pose des problèmes tant empiriques que théoriques, comme l'attestent le contenu des différents recueils ou dictionnaires spécialisés ou le fait que nombre de proverbes ont un auteur identifié et attesté.

A quoi s'ajoute le fait qu'un énoncé premier d'un signataire connu, dès l'instant où il se proverbialise, change de statut énonciatif selon qu'il appartient à son texte d'origine où fonctionne comme une «phrase détachée » (D. Maingueneau, 2012), entrée dans l'usage à force d'être citée.

VALERE [...] il faut que la frugalité règne dans les repas qu'on donne ; et que suivant le dire d'un ancien, il faut manger pour vivre et non pas vivre pour manger. $[\ldots]$

HARPAGON Qui est le grand homme qui a dit cela?

VALERE Je me souviens pas maintenant de son nom.

(Molière, L'Avare, Acte III, scène 1, Gallimard, «Bibliothèque de La Pléiade », tome 2, p. 548)

Heureusement, on dispose, aujourd'hui, d'un nombre appréciable d'études et de tentatives de définition des proverbes (J.-C. Anscombre (1994, 2000, 2011), G. Kleiber (2000), L. Perrin (2000, 2011, 2012), I. Tamba (2011) etc.) sur lesquelles nous allons nous appuyer. Précisons, cependant, que nous nous intéresserons moins aux spécificités internes des énoncés parémiques (caractéristiques formelles et sémantiques communes aux formes figées) et à leurs sous-espèces dont les proverbes, qu' aux usages discursifs des proverbes dans les fictions dramatiques. 
Qu'ils soient attestés ou inédits les proverbes ne sont pas rares dans les textes théâtraux.

Soit l'exemple suivant extrait d'une scène où l'on voit des passants regardant la police en train de négocier la reddition d'un homme qui menace de tuer une femme et son enfant :

UN HOMME.- $-[\ldots]$ le type est fichu.

UNE FEMME. - La femme aussi, sans doute.

UN HOMME.- On ne fait pas d'omelettes sans casser des æufs. [souligné par nous].

(B.-M. Koltès, Roberto Zucco, Les Éditions de Minuit, p. 62)

Énoncé concis, utilisant un présent dit générique et formulant un principe général dans le but de rendre compte d'une situation particulière, cette phrase possède bien les attributs majeurs d'une parole proverbiale. Plus précisément, le locuteur anonyme a recours à une sorte de raisonnement qui lui permet de confirmer la conclusion (Q) quant à l'issue fatale, en s'appuyant sur l'argument (P) en forme de loi d'évidence.

Du fait que les proverbes énoncent une «généralité intemporelle », ils possèdent des propriétés linguistiques que vérifient de nombreux tests que nous n'allons pas détailler. Retenons simplement qu'ils ne peuvent pas servir une énonciation événementielle ( Ce jour là, on ne fit pas d'omelettes sans casser des œufs » passe difficilement, à la différence de «Ce jour-là on a pas fait dans la dentelle » qui est une expression idiomatique mais pas générique). De même, le proverbe résiste à l'introduction par un déictique qu'il soit temporel $\left(A u^{-}\right.$ jourd 'hui, demain... on ne fait / on ne fera pas d'omelettes sans casser des œufs) ou spatial («A New-York... on ne fait pas d'omelettes sans casser des œufs »). Sauf à déproverbialiser l'énoncé proverbial et à le transformer en phrase idiomatique, bien sûr. Il est de même tout aussi possible de proverbialiser une phrase idiomatique événementielle ( $C$ 'est toujours la dernière goutte qui fait déborder le vase). Retenons aussi que les proverbes, bien qu'ils permettent de qualifier une situation, dans la mesure où ils sont des stéréotypes doxaux, sont difficilement compatibles avec une expression qui introduit un jugement individuel ou un point de vue personnel (Il est difficile d'accepter : "Pour ma part, il me semble, Je trouve, je crois, ...que l'on ne fait pas d'omelettes sans casser des œufs ». Ces expressions sont acceptables, par contre, quand elles servent à donner mon point de vue sur la validité du proverbe. De même, un bon test pour distinguer les proverbes des phrases idiomatiques singularisantes consiste à essayer d'y insérer « cette fois» ou «pour une fois ». Autant ce marqueur singularisant passe avec une phrase idiomatique ( Cette fois la coupe est pleine»; "Cette fois c'est la goutte qui fait déborder le vase »...), autant la manipulation est difficile avec les proverbes ( Cettefois, on ne fait pas d'omelettes sans casser des œufs »). Inversement, en tant que phrases génériques, les proverbes se combinent aisément avec « toujours », « généralement», « souvent» ou « jamais « et « rarement» quand ils sont à la forme négative («On a toujours ... souvent besoin d'un plus petit que soi »; «On fait rarement d'omelettes sans casser des œufs »).

Pour aller plus avant dans l'analyse des phrases proverbiales, nous dirons que ce sont des phrases génériques, qu'on appelle aussi typifiantes. Elles définissent un type au sens où elles présentent une propriété comme typique d'une classe ou 
d'un spécimen. Parmi les phrases typifiantes, on distingue celles qui sont vraies a priori car elles portent sur une propriété consensuelle mais pouvant contenir des exceptions qui confirment la règle. (ex. les chats n'aiment pas les chiens). Ce qu'atteste l'expression «Être comme chien et chat». Les typifiantes a priori s'opposent aux phrases analytiques comme « les chats sont des animaux » qui portent sur l'ensemble des individus d'une classe et ne tolèrent aucun contreexemple. Elles se distinguent aussi des phrases typifiantes dîtes locales (« les chats sont fourbes ") qui énoncent des propriétés plus discutables, voire contestables, que seul le locuteur ou un nombre réduit de locuteurs considèrent comme typique. Il est indéniable que les proverbes sont des phrases typifiantes et non pas des analytiques. L'existence d'un chat qui aime un chien n'invalide pas l'expression «Être comme chien et chat ». Quant à savoir si les proverbes sont des phrases typifiantes locales ou a priori, la question est plus délicate et nous n'entrerons pas dans les débats à ce sujet. Disons simplement que la vérité subjective exprimée par les proverbes ne tient pas tant à l'énonciation effective du locuteur, comme celle des typifiantes locales, mais entend s'appuyer sur un consensus préétabli, comme celle des phrases typifiantes a priori. Cela dit, cette vérité perçue comme consensuelle n'est pas due à un savoir établi sur le modèle d'une loi scientifique mais au fait qu'elle renvoie par convention, en vertu du caractère de dénomination que possèdent les proverbes, à une multitude d'énonciations préalables de la phrase même qu'ils réalisent. C'est cette force citative des proverbes qui leur confère une valeur générique et le pouvoir de dénommer un concept général associé à ses multiples contextes d'énonciations antérieures. C'est pourquoi le proverbe fait partie du vocabulaire au même titre que les mots ou les locutions de la langue. On comprend alors que les proverbes sont certes de nature idiomatique tout comme les expressions du type "Un ange passe » ou « Jeter l'éponge »... mais que contrairement à ces dernières, ils ne sont pas épisodiques.

Alors que les phrases idiomatiques ne prennent leur sens qu'en relation avec une situation communicationnelle donnée, les proverbes ont une valeur générique hors contexte. Comme l'affirme G. Kleiber (1989), les phrases génériques et les proverbes « constituent des régularités structurantes et non des assertions particulières ». Ils sont pourvus d'une sens généralisant, typifiant, relatif sinon à une loi, du moins à une règle de comportement ou de raisonnement.

ADRIEN.- Mathilde tu dors ? [...] Mes amis m'ont tout à fait lâché, je crois. À moins que tu y sois pour quelque chose. C'est probable; il n'y a pas de fumée sans $f e u$. [souligné par nous]

(B.-M. Koltès, Le Retour au désert, Les Éditions de Minuit, p. 61)

Ajoutons, pour clore la réflexion sur le statut de la parole proverbiale, que des formes de consensus existent aussi quant aux conditions requises à la proverbialisation (concision, autonomie, didacticité, fixité, fréquence d'emploi...). En ce sens, on trouve dans les pièces de théâtre des proverbes qui sont attestés mais bien d'autres aussi qui possèdent, plus ou moins, les qualités nécessaires pour pouvoir se proverbialiser pour peu qu'ils se mettent un jour à circuler au sein d'une communauté discursive et que s'efface la référence à un auteur particulier. Qu'on en juge, à partir de quelques exemples extraits de L'École des femmes de Molière, du Mariage de Figaro de Beaumarchais, de Combat de nègre et de chiens de Koltès et de Rhinocéros de Ionesco. 
Molière :

CHRYSALDE

Oui ; mais qui rit d'autrui

Doit craindre qu'en revanche on rit aussi de lui.

(Molière, L'École des femmes, Euvres complètes, Gallimard, «Bibliothèque de La Pléiade », volume 1, p. 547)

ARNOLPHE.

Épouser une sotte est pour n'être point sot.

$[\ldots]$

Et femme qui compose en sait plus qu'il ne faut.

(Molière, L'École des femmes, Euvres complètes, Gallimard, «Bibliothèque de La Pléiade », volume 1, p. 548)

Beaumarchais :

\section{BRID'OISON}

[...] Tel qui rit d'un juge en habit court, qui-i tremble au seul aspect d'un procureur en robe. La-a forme, la-a forme!

(Beaumarchais, Le Mariage de Figaro, Garnier Classique, p. 268)

FIGARO

Oh! que oui. Depuis qu'on a remarqué qu'avec le temps vieilles folies deviennent sagesse, et qu'anciens petits mensonges assez mal plantés ont produit de grosses vérités $[\ldots]$

(Beaumarchais, Le Mariage de Figaro, Garnier Classique, p. 286)

Koltès :

ALBOURI.- Souvent, les petites gens veulent une petite chose

(Koltès, Combat de nègre et de chiens, Les Éditions de Minuit, p. 31)

HORN.- Il vaut mieux exterminer le renard que de faire des sermons à la poule.

(Koltès, Combat de nègre et de chiens, Les Éditions de Minuit, p. 100)

CAL.- D'ailleurs, quand on connaît l'odeur, on connaît les gens...

(Koltès, Combat de nègre et de chiens, Les Éditions de Minuit, p. 38)

Ionesco :

Les braves hommes font les bons rhinocéros.

(Ionesco, Rhinocéros, Acte III, Gallimard, Folio, p. 207)

\section{2. Énonciation proverbiale et dialogisme}

Des recherches qui tiennent compte des potentialités énonciatives des proverbes, il ressort que le producteur d'une phrase sentencieuse rapportée, comme son interprète, ont plus ou moins conscience d'être en présence d'une hétérogénéité énonciative, d'un écho de forme et de contenu par l'intermédiaire d'une re-énonciation. Comme l'écrivait A.-G. Greimas (1970) : 
« [...] on a l'impression que le locuteur abandonne volontairement sa voix et en emprunte une autre pour proférer un segment de la parole qui ne lui appartient pas en propre, qu'il ne fait que citer $»$.

Ce qui implique de statuer sur la qualité de l'énonciateur premier et de décrire les modes d'insertion du proverbe dans la texture du discours second.

\subsection{Qualité de l'énonciateur premier}

Les notions de « sagesse des nations » ou de « sagesse populaire » n'étant pas des concepts linguistiques, J.-C. Anscombre (1994) propose de « donner une consistance linguistique à l'idée que l'auteur d'un proverbe est quelque chose comme une conscience linguistique collective. [...] celui qui énonce un proverbe, s'il est bien le locuteur du proverbe, n'est pas l'auteur de ce proverbe; en termes polyphoniques, il n'est pas l'énonciateur du principe qui lui est attaché. C'est lui en revanche qui endosse la responsabilité de déclarer ce principe applicable hic et nunc. La somme des proverbes est, de ce point de vue, comparable au corps des lois et le locuteur d'un proverbe est comparable à l'avocat qui utilise une loi, il n'est pas l'auteur de la loi, cet auteur étant la justice (à vocation universelle). En revanche, il est de la responsabilité de l'avocat de choisir de s'appuyer sur telle loi dans telle situation spécifique ».

A l'intérieur de la classe des phrases génériques, les proverbes sont des énoncés « exemplaires » au sens où ils servent d'appui en forme d'argument à une assertion. Ils ont, de ce fait, la propriété fonctionnelle de permettre la caractérisation d'un type de situation humaine - au niveau des personnages ou des lecteurs-spectateurs - par l'intermédiaire du recours dialogique à un savoir ou un contenu doxique supposés partagés et servant de garant.

OLMEDA. Venons au fait. Don Alvero, vous que nous nommons si respectueusement et si affectueusement « le Maître de Santiago », ne pensez-vous pas qu'il y aurait honneur pour vous à nous accompagner aux Indes? Vous connaissez le proverbe : «Il y a toujours une croisade en Espagne. ». La nouvelle croisade est là.

(Montherlant, Le Maître de Santiago, Acte I, scène 4, Livre de Poche, $\mathrm{n}^{\circ} 1172$, p. 42)

Dans le cadre d'une analyse polyphonique, on peut dire que le locuteur du proverbe accepte le principe associé au contenu de l'énonciation à laquelle il fait écho mais le fait sous couvert d'une autre énonciateur « $\mathrm{ON}$ » qui est le garant de la vérité du proverbe. Celui qui énonce le proverbe présente son assertion comme un écho à la multitude des énonciations antérieures de ce même proverbe. Ce que souligne L. Perrin (2012) : «Plutôt que de raisonner par l'autorité d'un auteur individuel, les proverbes se fondent sur l'autorité d'une voix collective, dont la force autoritaire repose précisément sur le caractère à la fois ancien et partagé [...] Énoncer un proverbe, c'est non seulement raisonner par autorité, mais c'est avant tout faire résonner la voix collective ancestrale à laquelle la phrase fait écho. ».

En ce sens, comme l'écrit A. Berrendonner (1981): « [...] tout proverbe ou énoncé gnomique se donne, contrairement aux énoncés de simple information 
("Pierre est tombé malade"), comme performé sur le mode du complexe illocutoire "Je conviens que...". Sa structure stéréotypée d'“expression toute faite"» [...] est donc l'indice que son contenu fait l'objet d'une présupposition de ON-vérité ».

Le ON du prédicat «ON-vrai » qui le fonde coïncide, en fait, avec l'ensemble des locuteurs de la langue dont la compétence lexicale inclut la connaissance du sens des proverbes ainsi que l'acceptation de leur autorité doxique.

C'est pourquoi, on peut dire qu'une des fonctions des proverbes est d'indicier le système de valeurs des personnages et de contribuer ainsi à leur description. Ce qu'illustrent les exemples suivants extraits de Combat de nègre et de chiens, de B.-M. Koltès :

A Horn qui le menace, Alboury rétorque :

ALBOURY.- Un scorpion qu'on tue revient toujours. (p. 29)

Le même Horn conseille à Cal :

HORN.- Il vaut mieux exterminer le renard que de faire des sermons à la poule. (p. 100)

Quant à Cal, sa parole proverbiale révèle son idéologie.

CAL._- D'ailleurs, quand on connaît l'odeur, on connaît les gens... (p. 38)

CAL.- Crachats de boubous sont menace pour nous. (p. 78)

\subsection{Modes d'insertion du proverbe}

Il est d'usage d'introduire le proverbe dans le texte citant par des expressions du type « comme on dit», " comme dit le proverbe », « comme le dit la sagesse populaire » ou en référence à un énonciateur collectif et anonyme.

MADAME PERNELLE [...] Et vous, n'y touchez pas, tant vous semblez doucette! Mais il est, comme on dit, pire eau que l'eau qui dort.

(Molière, Tartuffe, Acte I, scène 1, Gallimard, " Bibliothèque de La Pléiade ", tome, 1 page 896 )

ALVARO. [...] La trahison est toujours sous notre toit, et pas seulement à la cuisine, comme on le dit. (Appelant:) Mariana.

(Montherlant, Le Maître de Santiago, Acte II, scène 1, Livre de Poche, ${ }^{\circ}{ }^{1172}$, p. 90)

\section{SGANARELLE}

Hé ! mon Dieu, monsieur, ne soyez point en peine ; je vous réponds que je ferai aussi bien mourir une personne qu'aucun médecin qui est dans la ville. On dit un proverbe, d'ordinaire : Après la mort le médecin; mais vous verrez que si je m'en mêle on dira : Après le médecin, gare la mort!

(Molière, Le Médecin volant, scène 1, Euvres complètes, Éditions du Seuil, p. 41) 
BAZILE

[...] Chérubin! Chérubin! vous lui causerez des chagrins ! Tant va la cruche à l'eau, qu'à la fin...

FIGARO

Ah ! voilà notre imbécile avec ses vieux proverbes ! Eh bien, pédant, que dit la sagesse des nations? Tant va la cruche à l'eau, qu'à la fin...

(Beaumarchais, Le Mariage de Figaro, Acte I, scène 11, Classique Garnier, p. 209)

Le locuteur/énonciateur d'un proverbe est détenteur non d'un savoir rationnel ("Les chats sont des animaux, comme on dit» passe difficilement) mais de croyances concernant la nature des choses et utilisées sur le mode de l'analogie.

À Horn qui lui dit que c'est une chose insensée de vouloir prendre une femme, compte tenu de son âge, Alboury lui répond :

ALBOURY.- Les femmes ne sont pas des choses insensées. Elles disent d'ailleurs que c'est dans les vielles marmites qu'on fait la meilleure soupe. Elles ont leurs mots à elles, mais c'est très honorable pour vous. [souligné par nous]

(B.-M. Koltès, Combat de nègre et de chiens, Les Éditions de Minuit, p. 13)

Toutefois, il est possible, et c'est plutôt le cas chez Koltès, d'insérer le proverbe sans aucun élément introducteur, ce qui renforce la validité universelle de la formule et l'indexe comme un stéréotype d'opinion.

ADRIEN.- Mathilde tu dors ? [...] Mes amis m'ont tout à fait lâché, je crois. À moins que tu y sois pour quelque chose. C'est probable; il n'y a pas de fumée sans $\mathrm{feu}$. [souligné par nous]

(B.-M. Koltès, Le Retour au désert, Les Éditions de Minuit, p. 61)

Sa notoriété seule, dans ce cas, garantit son statut de citation. C'est cette dernière qui permet d'expliquer qu'une phrase d'auteur peut se proverbialiser. Dans un premier temps (intertextualité explicite), on cite l'énoncé en référence à son auteur et en l'appliquant à une situation particulière. Par la suite, c'est un phénomène de circulation des discours au sein des communautés langagières, à force d'être répété, l'énoncé s'attribue la capacité à caractériser une classe générique de situation sans que l'on ait besoin de faire référence à son auteur original. Ce phénomène d'implicitation de la relation d'intertextualité est confirmé par le fait que certains proverbes sont tellement inscrits dans la mémoire collective qu'il suffit de n'en citer qu'une partie ( « Au royaume des aveugles ... les borgnes sont rois. »; "Rien ne sert de courir... il faut partir à point »). Dans notre corpus théâtral, les phrases sentencieuses font l'objet d'un double mouvement circulatoire : importation à partir de sources plus ou moins identifiables (citations tirées d'œuvres et d'auteurs anciens ou d'ouvrages qui font autorité tels que la Bible) ou sur la base de l'anonymat; exportation vers le discours commun d'une phrase extraite d'une œuvre dramatique qui, à force d'être répétée, acquiert le statut de pensée anonyme. Corneille est à cet égard passé maître en la matière, comme le rappelle D. Maingueneau (1992, 2012), qui écrit :

«On comprend le lien privilégié qui s'établit entre ces surassertions sentencieuses et l'héroïsme cornélien. Le héros n'est pas seulement un personnage qui accomplit des actes exceptionnels :c'est aussi quelqu'un qui énonce en héros [...] Il 
n'accomplit pas des actes ordinaires mais des actes qu'accomplit l'homme par excellence, que dans cette situation tout homme pleinement homme se doit d'accomplir. En proférant de telles maximes, il réalise donc discursivement l'exemplarité héroïque : il profère sa parole sur deux registres à a fois, celui du JE et celui du ON, il fait entendre l'universalité du ON dans la singularité du JE. Il est celui qui se détache du commun des mortels et dont le détachement se manifeste par la profération d'énoncés qui seront détachés par ceux qui l'admirent. »

\section{Fonctions textuelles des proverbes}

Du fait que le locuteur d'un proverbe n'en n'est pas l'auteur mais l'utilisateur d'une dénomination inscrite dans la langue, il importe de se demander, dans le cadre de la dialogie interne des échanges entre les personnages, quel est le but communicationnel poursuivi par celui qui utilise un proverbe.

L'hypothèse que l'on peut faire, ici, est que le proverbe, en discours, appartient à un dispositif argumentatif et qu'il existe des points communs entre le topos et le proverbe.

C'est qu'un proverbe n'a pas pour fonction de fournir une information par lui-même mais sert à étayer une argumentation ou à concrétiser un raisonnement explicatif en servant de cadre et de garant à une argumentation. Ce dont E. Benveniste (1966) avait rendu compte quand il constatait que chez Homère « la phrase nominale apparaît seulement dans les discours, non dans les parties narratives ou descriptives, et qu'elle exprime des assertions de valeur permanente, non des situations occasionnelles. [...] L'énonciation ainsi formulée, à cause même du caractère permanent de son contenu, est apte à servir de référence, de justification, quand on veut créer une conviction. [...] C'est pourquoi la phrase nominale convient si bien à ces énonciations où elle tend d'ailleurs à se confiner, sentences ou proverbes, après avoir connu plus de souplesse ».

Reprenons notre premier exemple :

UN HOMME.- $[\ldots]$ le type est fichu.

UNE FEMME. - La femme aussi, sans doute.

UN HOMME.- On ne fait pas d'omelettes sans casser des œufs.

(B.-M. Koltès, Roberto Zucco, Les Éditions de Minuit, p. 62)

C'est parce qu'il existe un topos bien connu du type On ne fait pas d'omelettes sans casser des oufs que l'homme peut inférer de la situation à laquelle il assiste que «le type est fichu».

La similitude entre les proverbes et les topoï tient au fait qu'il s'agit, dans les deux cas, de principes généraux qui font l'objet d'un consensus au sein d'une communauté discursive plus ou moins étendue, principes présentés comme ayant force de loi et servant d'appui au raisonnement.

Ce rôle du proverbe comme «moteur argumentatif validant le raisonnement » (J.-C. Anscombre, 1995) est confirmé par le fait qu'il ne peut pas avoir le statut d'une conclusion tirée d'un raisonnement puisque le contenu de savoir qu'il contient est déjà connu. Il s'en suit qu'il est difficile d'accepter : "Par conséquent, l'on ne fait pas d'omelettes sans casser des œufs ». Inversement, une ex- 
pression comme «Étant donné que... », qui sert à introduire les prémisses d'un discours, se combine avec un proverbe, pour les mêmes raisons «Étant donné que l'on ne fait pas d'omelettes sans casser des œufs...».

On sait combien abondent dans l'œuvre de Koltès les échanges verbaux qui relèvent de l'argumentation, ce dont le dramaturge avait conscience, lui qui écrivait : «Un dialogue ne vient jamais naturellement. Je verrais volontiers deux personnes face à face, l'une exposer son affaire et l'autre prendre le relais. Le texte de la seconde personne ne pourra venir que d'une impulsion première. Pour moi, un vrai dialogue est toujours une argumentation, comme en faisaient les philosophes mais détournée. " (Une part de ma vie, p. 23). C'est ainsi que S. Hage (2011) a montré que de nombreuses interactions entre les personnages reposaient sur des procédés rhétoriques hérités des genres de discours antiques, que ce soient les controverses apparentées au cadre judiciaire dans Combat de nègre et de chiens et dans Le Retour au désert ou les processus sophistiques qu'utilisent Fak dans son échange avec Claire dans Quai ouest. On peut y ajouter les proverbes en forme d'argument dont on sait la fonction que leur accordait Aristote dans sa Rhétorique quand il écrivait que « certains proverbes sont aussi des maximes » (Livre II, 21, « Des maximes »).

Il reste à mesurer si cet usage des proverbes est socialement indifférencié ou s'il peut être considéré comme un " signum » de classe. À regarder les œuvres dramatiques du passé on aurait tendance à dire que l'usage des proverbes à l'époque classique était un fait générique (il est à la comédie ce que la maxime est à la tragédie) et un marqueur de classe puisque ce sont essentiellement les personnages populaires (J.-J. Tatin, 1983) qui en font usage sur le mode sérieux. Les exemples abondent chez Molière où ce sont les valets et les suivantes qui pratiquent le discours proverbial, comme l'atteste la tirade ridicule de Sganarelle :

SGANARALLE : [...] Sachez, Monsieur, que tant va la cruche à l'eau qu'enfin elle se brise ; et comme dit fort bien cet auteur que je ne connais pas, l'homme est en ce monde ainsi que l'oiseau sur la branche ; la branche est attachée à l'arbre ; qui s'attache à l'arbre suit de bons préceptes [...].

(Molière, Dom Juan ou le Festin de Pierre, Gallimard, "Bibliothèque de La Pléiade ", tome 2, p. 82)

Cette fonction diégétique de production d'effets de stéréotypie sociale se retrouve dans la production dramatique contemporaine.

C'est ainsi, pour ne prendre qu'un exemple, qu'un auteur contemporain tel que Lemahieu caractérise les personnages au statut d'ouvrier en recourant à des locutions sentencieuse utilisées comme des connotateurs de réalité socio-discursive.

L'ONCLE.- Après l'effort le réconfort (Lemahieu, Usinage, Théâtre I, Domens, p. 181)

L'ONCLE.- Quand y en a plus y en a encore (Lemahieu, idem, p. 181)

LE COPAIN.—L'heure c'est l'heure avant l'heure c'est pas l'heure (Lemahieu, idem, p. 178) 
Il est à souligner que chez Koltès ce sont surtout les personnages qui ont un statut de marginaux sociaux ou les représentants d'une altérité culturelle qui font usage de formes sentencieuses, qu'elles soient attestées, fassent écho à des énoncés qu'elles imitent ou forgées de toute pièce.

ALBOURI.- Souvent, les petites gens veulent une petite chose.

(B.-M. Koltès, Combat de nègre et de chiens, Les Éditions de Minuit, p. 31)

ALBOURY.- Je leur dirai cela en ramenant le corps : pas de casque, pas un sou.

(B.-M. Koltès, Combat de nègre et de chiens, Les Éditions de Minuit, p. 27)

LE BALEZE.- Un homme ne doit pas se laisser mordre deux fois par le même chien.

(B.-M. Koltès, Roberto Zucco, Les Éditions de Minuit, p. 48)

Il arrive aussi, comme on peut le voir pour les titres de certains articles de presse (Sullet-Nylander, F., 2002 ; Leroy, S., 2005) que les formes sentencieuses (proverbes, citations...) existantes soient reformulées.

\section{BAZILE}

Vous aviez l'air d'accord ; je n'y entendais rien ; et dans les cas difficiles à juger, une bourse d'or me paraît toujours un argument sans réplique. Et puis, comme dit le proverbe, ce qui est bon à prendre...

$$
\text { BARTHOLO }
$$

J'entends, est bon...

\section{BAZILE}

A garder.

\section{BARTHOLO}

$\mathrm{Ah} ! \mathrm{Ah} !$

BAZILE

Oui, j'ai arrange comme cela plusieurs petits proverbs avec des variations ; mais allons au fait ; à quoi vous arrêtez-vous?

(Beaumarchais, Le Mariage de Figaro, Acte IV, scène 1, Classique Garnier, p. 113)

On est bien en présence de ce qu'A. Grésillon et D. Maingueneau (1984) appellent le « détournement de proverbes ». Ils définissent ce dernier comme étant un procédé discursif qui «consiste à produire un énoncé possédant les marques linguistiques de l'énonciation proverbiale mais qui n'appartient pas au stock des proverbs reconnus » et précisent que cette "pratique peut être mise en œuvre sous deux régimes très différents, l'un ludique, l'autre miltant ».

Que 1'enjeu du détournement soit de type ludique ou critique, il s'agit d'un mode particulier de dialogisme intertextuel puisqu'il présuppose pour être apprécié comme tel que le récepteur connaisse l'énoncé originel.

Les modes possibles de détournement de phrases figées sont nombreux et variés, comme l'atteste la typologie des formes figées proposée par F. Rastier (1997). Compte tenu de notre corpus théâtral restreint, nous nous limitons à trois exemples.

— Allusion à un énoncé déposé dans la mémoire culturelle

ESTRAGON.- On ne descend pas deux fois dans le même pus.

(S. Beckett, En attendant Godot, Les Éditions de Minuit, p. 84) 
L'écho à Héraclite crée par la similitude syntaxique exploite la réflexion de ce dernier sur la condition humaine mais sur le mode pessimiste. En remplaçant la lexie initiale par « descendre dans un puits », Beckett conserve l'isotopie aquatique tout en substituant à la fluidité du «fleuve » la stagnation du «puits », qui est elle-même renforcée par le jeu homophonique avec le «pus».

- Reprise modifiée d'un proverbe.

BAZILE

[...] Chérubin ! Chérubin! vous lui causerez des chagrins ! Tant va la cruche à l'eau, qu'à la fin...

FIGARO

Ah! voilà notre imbécile avec ses vieux proverbes ! Eh bien, pédant, que dit la sagesse des nations? Tant va la cruche à l'eau, qu'à la fin...

BAZILE

Elle s'emplit.

FIGARO

Pas si bête, pourtant, pas si bête!

(Beaumarchais, Le Mariage de Figaro, Acte I, scène 11, Classique Garnier, p. 209)

Facilement reconnaissable puisque repris à l'identique, à l'exception du verbe, le sens du proverbe change cependant totalement, remplacé qu'il est par une isotopie sexuelle tout à fait pertinente dans le contexte de la scène.

— Révision d'une source textuelle qui fait autorité.

VICTOR.- Et le fruit de votre entaille est béni.

LILI. - D'abord, c'est le fruit de vos entrailles qu'il faut dire.

VICTOR.- Peut-être, mais c'est moins imagé.

(C. Vitrac, Victor ou les enfants au pouvoir, Folio, p. 33)

En remplaçant « entraille » par « entaille», le détournement est d'autant plus réussi que la modification est minimale sur le plan du signifiant mais fortement subversive au niveau du signifié. En effet, il s'agit de contester l'interprétation théologique en faisant une allusion triviale à la réalité corporelle. Ce n'est pas un hasard si Vitrac a été compagnon de route du surréalisme dont on sait que Breton et Éluard, auteurs de 152 proverbes mis au goût du jour, ont entrepris de dénoncer l'assujettissement idéologique de la parole proverbiale

\section{Conclusion}

Pour résumer notre propos, nous pensons avoir montré, dans la première partie de notre article, (Statut de la parole proverbiale), qu'un proverbe peut se définir sur la base de critères différents : syntaxiques (syntaxe elliptique, emploi du déterminant zéro), sémantiques (énoncé doxal, dénommant une situation générique), énonciatifs (forme citative qui respecte un ON-DIT explicite ou implicite, présent générique ou réalisation zéro), formels (structures brèves), rythmiques (souvent formule rythmée voire rimique, symétries phoniques et prosodiques), pragmatiques (enjeu argumentatif sur la base d'une relation du type « $\mathrm{P}$ est un argument pour $\mathrm{Q} »)$. Ajoutons que le proverbe constitue un énoncé auto- 
nome mais ne fonctionne qu'inscrit dans un discours qui l'intègre intertextuellement.

C'est pourquoi, dans notre deuxième partie (Énonciation proverbiale et dialogisme), en décrivant les modes de leur insertion dans la texture du texte dramatique, nous avons montré que les proverbes sont à classer parmi les phénomènes de « mention ». Énonciation faisant écho à une chaîne de locuteurs, le proverbe acquiert ainsi une autorité investie par l'usage. Cette dissociation polyphonique fait que le locuteur de l'énoncé parémique n'est pas son auteur originel mais le responsable de son réemploi et de l'application à une situation donnée de la vérité générale que formule l'énoncé.

Ce cadre interdiscursif et polyphonique étant rappelé, nous avons cherché, dans la troisième partie (Fonctions textuelles des proverbes) à analyser les fonctions des phrases sentencieuses dans les dialogues théâtraux : fonction sapientielle et doxale de transmission de vérités sur le monde des humains dont il revient au lecteur d'en mesurer la portée et de savoir si elles sont attribuables aux seuls personnages ou assumées par l'auteur; fonction diégétique de description indirecte des personnages et de leur système de valeur; stratégie argumentative déployée par les personnages puisque les proverbes sont des énoncés «exemplaires » au sens où ils servent d'appui, en forme d'argument, à une assertion ; fonction ludique ou satirique des détournements de proverbes. À quoi s'ajoute le fait, sauf à contester polémiquement leur contenu, que le recours aux proverbes est destiné à laisser l'autre dans l'impossibilité de répliquer.

Nous dirons, pour conclure, que les énoncés sentencieux interpellent le lecteur ou le spectateur sur le mode de la reconnaissance et du partage des normes et des valeurs sociales et culturelles ou sur le mode de la mise à distance, voire de leur critique. 


\section{Bibliographie}

\section{Textes théoriques}

ANSCOMBRE, J.-C. (1994), «Proverbes et formes proverbiales : valeur évidentielle et argumentative », Langue Française, n ${ }^{\circ}$ 102, 95-107.

— (1995), « La théorie des topoï : sémantique ou rhétorique? », Hermès, $\mathrm{n}^{\circ}$ $15,185-198$.

- (2000), «Parole proverbiale et structures métriques », Langages, $\mathrm{n}^{\circ} 139$, 6-26.

- (2011), «Figement, idiomaticité et matrices lexicales » in Le figement linguistique : la parole entravée, textes réunis par J.-C. Anscombre et S. Mejri, H. Champion, 17-40.

ARNAUD, P.J.L. (1991), « Réflexion sur le proverbe », Cahiers de lexicologie, LIX-2, 6-27.

Benveniste, E. (1966), «La phrase nominale », in Problèmes de linguistique générale, Gallimard, 151-167.

BERRENDONNER, A. (1981), «Le fantôme de la vérité », in Eléments de pragmatique linguistique, Les Éditions de Minuit, 35-73.

GreimAS, A.-J. (1970), «Les proverbes et les dictons » in Du sens, Éditions du Seuil, 309.

GréSillon, A., Maingueneau, D. (1984), « Polyphonie, proverbe et détournement, ou un proverbe peut en cacher un autre », Langages, $\mathrm{n}^{\circ} 73$, Larousse, 112-125.

HAGE, S. (2011), « L'argumentation koltésienne dans Quai ouest, entre dysfonctionnement et sophistique », in Bernard-Marie Koltès, Textes et Contextes, A. Petitjean, (éd), « Recherches Textuelles », ${ }^{\circ} 10$, Université Paul-Verlaine-Metz, 363-380.

KLEIBER, G. (1989), « Sur la définition des proverbes », Phraséologie contrastive, G. Greciano, (Ed), Recherches Germaniques, $\mathrm{n}^{\circ} 2$, Université de Sciences Humaines de Strasbourg, 233-253.

- (2000), « Sur le sens des proverbes », in Langages, $\mathrm{n}^{\circ} 139,39-58$.

LEROY, S. (2005), « Le détournement dans les titres de presse : un marquage dialogique ? », in Bres, J., P.P. Haillet, S. Mellet, H. Nølke, L. Rosier (sous la dir.) : Dialogisme et polyphonie: Approches linguistiques. Actes du colloque de CERISY, Bruxelles, De Boeck \& Larcier-Duculot, 201-214.

MAINGUENEAU, D. (1992), «Un problème cornélien : la maxime », Etudes littéraire, vol. 25, $\mathrm{n}^{\circ} 1 / 2$, Québec, 11-22.

- (2012), Les phrases sans texte, A. Colin.

PERRIN, L.(2000), « Remarques sur la dimension générique et sur la dimension dénominative des proverbes », Langages, $\mathrm{n}^{\circ} 139,69-80$.

- (2011), «Figement, énonciation et lexicalisation citative » in Le figement linguistique : la parole entravée, textes réunis par J.-C. Anscombre et S. Mejri, H. Champion, pp. 81-94.

- (2012), "L'énonciation des proverbes », dans J.-C. Anscombre, B. Darbord et A. Oddo, La parole exemplaire. Introduction à une étude linguistique des proverbes, Armand Colin, 53-66. 
RASTIER, F. (1997), «Défigements sémantiques en contexte » in La locution, entre langue et usages, M. Martins-Baltar (éd), Paris, Orphrys, 305-329.

Sullet-Nylander, F. (2002), « Titres de presse et polyphonie », Romansk Forum $16,767-77$.

TAMBA, I. (2011), «Sens figé :idiomes et proverbes » in Le figement linguistique: la parole entravée, textes réunis par J.-C. Anscombre et S. Mejri, Paris, H. Champion, 109-126.

TAtin, J-J. (1983), «Proverbes et voix du peuple », Revue des Sciences Humaines, Tome LXI, $\mathrm{n}^{\circ} 190,21-27$.

\section{Textes dramatiques}

Beaumarchais, Le Barbier de Séville, Classiques Garnier.

BeAumARCHAIs, Le Mariage de Figaro, Classiques Garnier.

BECKETT, S., En attendant Godot, Les Éditions de Minuit.

IONESCO, Rhinocéros, Gallimard, Folio.

LEMAHIEU, D. Usinage, Théâtre I, Domens.

Koltès, B.-M., Combat de nègre et de chiens, Les Éditions de Minuit.

Koltès, B.-M., Le Retour au désert, Les Éditions de Minuit.

Koltès, B.-M., Roberto Zucco, Les Éditions de Minuit.

MoliÈre, Le Médecin volant, Euvres complètes, Gallimard, "Bibliothèque de La Pléiade », tome 1.

MoliÈre, L'Avare, Euvres complètes, Gallimard, "Bibliothèque de La Pléiade », tome 2.

MoliÈre, Tartuffe, Euvres complètes, Gallimard, "Bibliothèque de La Pléiade », tome, 1.

MOLIÈRE, L'Ecole des femmes, Euvres complètes, Gallimard, « Bibliothèque de La Pléiade », tome, 1.

MOLIÈRE, Dom Juan ou le Festin de Pierre, Euvres complètes, Gallimard, « Bibliothèque de La Pléiade », tome 2 .

Montherlant, Le Maître de Santiago, Livre de poche, ${ }^{\circ} 1172$.

VITRAC, R. Victor ou les enfants au pouvoir, Folio. 